\title{
COMMENT
}

\section{A Reconsideration of Measures of Instability ${ }^{1}$}

\author{
CHWO-MING YU \\ Graduate School of Business Administration, University of Michigan, \\ Ann Arbor, Michigan 48109
}

Received September 22, 1985; revised March 3, 1986

Using three measures, namely, the standard deviation of the ratio of the deficit to GDP and the slope and standard error of the estimate of regressing this ratio on time, Brewer (1985) concludes that the developing countries' deficits, as a group, are not notably larger or more unstable than the industrial countries' deficits. This comment demonstrates that by using a more appropriate measure of instability, the developing countries as a group actually exhibit greater stability.

Variation over time is an indicator of instability. The greater the variatiol., the greater the instability. Because standard deviation is one measure of variation, it is often used as a measure of instability. In some cases, however, standard deviation may not be an appropriate measure to reflect instability. For a set of time-series observations, the underlying pattern demonstrated by them may be composed of two parts: a trend part which is predictable, and an uncertainty part which is unpredictable. Since the trend part can be predicted with much confidence and the uncertainty part cannot, we think that only the uncertainty part reflects the essence of instability. Because the calculation of standard deviation does not explicitly consider the trend part, we doubt the appropriateness of using the standard error to measure instability for time-series observations. Case 1 and case 2 in Table 1 are good examples. The mean is the same in the two cases, but the standard deviation of case 1 is higher than that of case 2 . This seems to suggest that case 1 is more unstable than case 2. But an examination of data in Table 1 shows that case 1 is more stable than case 2 since the former has a constant increase of amount $q$ for each time period. This points to the limitation of using standard deviation as the measure of instability. If the values of observation are steadily decreasing

\footnotetext{
' The author thanks the anonymous reviewers for their helpful comments.
} 
TABLE 1

ILLUSTRATIVE CASES

\begin{tabular}{lccc}
\hline & Case 1 & Case 2 & Case 3 \\
\hline Time period & & & \\
1 & $p$ & $p$ & $p$ \\
2 & $p+q$ & $p+2 q$ & $p+2 q$ \\
3 & $p+3 q$ & $p+3 q$ & $p+3 q$ \\
4 & $p+4 q$ & $p+3.5 q$ & $p+q$ \\
5 & $p+2 q$ & $p+2 q$ & $p+4 q$ \\
Means & $1.41 q$ & $1.22 q$ & $p+2 q$ \\
Standard deviations & $q^{a}$ & $0.65 q$ & $1.41 q$ \\
Regression coefficients & 0 & $1.04 q$ & $0.70 q$ \\
Standard error of estimates & & & $1.30 q$ \\
\hline
\end{tabular}

${ }^{a}$ Significant at $5 \%$ level.

or increasing over time, the standard deviation is not sensitive enough to reflect this phenomenon. In this situation, the standard error of the estimate of regressing observations on time is a preferred measure of instability.

There are two questions about the case of the standard error as a measure of instability. First, is this measure sensitive enough to differentiate between cases with the same set of observations but with different orderings, such as case 1 and case 3 in Table 1? As Table 1 shows, the regression coefficent for case 1 is significantly different from zero, and its standard error is less than that of case 3. This means that the standard error has differentiating power in this situation. However, the second question leads to a different conclusion. Suppose that the regression coefticient is not statistically different from zero. In this case, is the standard error still a preferred measure? Clearly not, because the standard error is calculated from the squared deviations between observed and predicted values. The predicted values are calculated by using the insignificant regression coefficient and thus tend to provide an inaccurate measure of variation. Therefore, in this situation, the standard error of the estimate is not an appropriate measure of instability.

Based on the above arguments, we propose a measure which can make up for all of these deficiencies. The measure, hereinafter called the new measure, is defined as

(1) standard error of the estimate/mean, which is appropriate if the regression coefficient is significant, and

(2) standard deviation/mean, which is appropriate otherwise. 
The 38 countries studied by Brewer (1985) are ranked according to their values on the three measures (Table 2). The higher the value of the measure of instability of one country compared to those of other countries, the lower its rank relative to other countries. Thus, the higher the rank, the greater the stability. For example, if the measure is standard deviation, and if the standard deviation of country A is higher than that of country B, then country B has a higher rank. In cases where several countries have the same value, the average rank is assigned to each of them. Ranks are then classified into seven categories: $1-5,6-10,11-15,16-20,21-25,26-30$, and over 30 . For example, countries with ranks between 1 to 5 belong to the first category. Comparing the results using standard deviation and standard error of estimate with those of the new measure, we find the following:

(1) The Spearman rank correlation coefficient is 0.91 for the standard deviation and the standard error of the estimate. The high association between the ranking of these two measures, coupled with the similar distribution of ranks in Table 2, indicates that these two measures reveal the same information: that the developing countries are not more unstable than the industrial countries. However, this conclusion is suspect because of the deficiencies associated with these two measures.

(2) The Spearman rank correlation coefficients are only -0.17 and 0.01 for the new measure and the standard deviation and for the new measure and the standard error of the estimate, respectively. These low correlation coefficients, which are not statistically significant at the $1 \%$ level, indicate that the new measure reveals different ordering of ranks than do the other two measures.

TABLE 2

DISTRIBUTION OF RANKS BY DIFFERENT MEASURES

\begin{tabular}{llllllll}
\hline & \multicolumn{7}{c}{ Category of ranks } \\
\cline { 2 - 8 } \multicolumn{1}{c}{ Measures } & $1-5$ & $6-10$ & $11-15$ & $16-20$ & $21-25$ & $26-30$ & Over 30 \\
\hline $\begin{array}{l}\text { Standard deviations } \\
\quad \text { Industrial }\end{array}$ & 3 & 3 & 2 & 2 & 1 & 4 & 4 \\
$\quad$ Developing & 1 & 3 & 4 & 2 & 4 & 1 & 4 \\
$\quad \begin{array}{l}\text { Standard error of estimates } \\
\quad \text { Industrial }\end{array}$ & 2 & 4 & 2 & 2 & 4 & 2 & 3 \\
$\quad$ Developing & 3 & 2 & 2 & 3 & 2 & 1 & 6 \\
$\quad$ New measures & & & & & & & \\
$\quad$ Industrial & 1 & 2 & 3 & 0 & 6 & 4 & 3 \\
$\quad$ Developing & 2 & 2 & 4 & 5 & 0 & 1 & 5 \\
\hline
\end{tabular}


(3) As in the case of the rankings, the distribution of ranks of the standard deviation and the standard error of the estimate is different from that of the new measure. Across the first four categories of ranks, which encompass rank 1 to rank 20, the new measure gives the developing countries a much greater share than it does for the industrial countries. Further, a similar result is found for the new measure across the first two categories of ranks, although it is not as strong. Therefore, the new measure shows that the developing countries, as a group, demonstrate more stability than do the industrial countries.

In conclusion, if we accept the new measure as more appropriate than the other two measures, then our analysis provides substantial support to Brewer's argument that the deficits of many developing countries have actually been small or stable relative to the deficits of many industrial countries over the period 1967-1981.

\section{REFERENCE}

Brewer, Thomas L., “A Comparative Analysis of the Fiscal Policies of Industrial and Developing Countries-Policy Instability and Governmental-Regime Instability." J. Comp. Econom. 9, 2:191-196, June 1985. 